\title{
SUPPURATIVE PERIARTHRITIS IN AN INFANT DUE TO THE SUIPESTIFER BACILLUS
}

\author{
BY \\ KATHARINE J. GUTHRIE, M.D. \\ (From the Department of Pathology of the Royal Hospital for Sick \\ Children, Glasgow)
}

Salmonella infections in infancy and early childhood show a marked tendency to invade the blood-stream and produce foci of suppuration in various organs and tissues. Certain strains appear to become localized in particular sites; thus b. enteritidis (Gaertner's bacillus) and the closely related b. dublin, frequently infect the meninges causing suppurative meningitis. B. suipestifer (the hog cholera bacillus), on the other hand, tends rather to produce lesions in bones and joints. The following case of suipestifer infection illustrates involvement of the periarticular tissues of the right shoulder joint.

\section{Case report}

The subject, a male infant aged seven months, was admitted to hospital on November 19, 1937. For the previous week he had been irritable, screaming when the right arm was moved. He did not use the arm above the elbow though able to move the fingers. Apart from a 'cold' during the previous two months he had always been healthy and the bowels had been regular. He had been breast fed. On examination the child was pale and had an upper respiratory infection. The right shoulder was fuller than the left and there was tenderness on attempting movement of the right arm. The upper part of the humerus also was sensitive to pressure. On $x$-ray examination the day after admission an arthritis of the right shoulder joint was diagnosed. Signs of broncho-pneumonia rapidly developed followed by nuchal rigidity and increased irritability. Lumbar puncture was performed on November 22, when the cerebro-spinal fluid was under slightly increased pressure. Microscopic examination, however, showed no noteworthy increase in cells and no organisms and cultures remained sterile. Death occurred on November 23, 1937.

Post-mortem findings. At autopsy exploration of the right shoulder joint revealed a collection of thick yellow pus round the joint chiefly on the anterior and inferior aspects. This was not confined to any of the related bursae. The joint capsule was intact and the joint itself was not inflamed and was free from effusion. No pus was found when the right femur was split lengthwise. The lungs showed pus in the bronchioles with patchy broncho-pneumonia of suppurative type at both bases. Nothing else of note was present in the thorax. There was no gross morbid change in the liver and kidneys, and the stomach and intestines were normal. The spleen was of septic type. Abundant pus was present in both middle ears. There was no meningitis and the brain substance was normal. 
Bacteriology. Films of the periarticular pus showed scanty Gram-negative coliform bacilli. Cultures made in broth yielded a pure growth of a Gramnegative actively motile bacillus, and on MacConkey plates the colonies were all of non-lactose-fermenting type. Several were subcultured and produced acid and gas in glucose, mannite and maltose. Lactose, saccharose and dulcite were not fermented and no indole was formed. These reactions suggested that the strain belonged to the Salmonella group and confirmation was obtained on testing with a polyvalent Salmonella serum (Oxford standards) which agglutinated it to titre. Pus from both ears also yielded a non-lactosefermenting growth similar in all respects to that obtained from the periarticular pus. Cultures were next tested against a paratyphosus B antiserum of high titre, but no agglutination occurred even in a dilution of 1100 . Other Salmonella antisera gave a negative result with the exception of paratyphosus $C$ antiserum (Oxford standards) which aggiutinated cultures practically to titre, thus suggesting that the organism might be a strain of $b$. suipestifer which belongs to the paratyphosus $\mathrm{C}$ group. The unknown Yorkhill culture was then further investigated along with Lister Institute strains of the two common suipestifer types-American and European (Kunzendorf). The series of biological tests to which the three cultures were subjected was the same as that employed by Guthrie and Montgomery (1939). All three strains gave similar fermentation reactions, rapidly producing acid and gas in sorbite, rhamnose and xylose and failing even after a week to ferment arabinose, trehalose and dextrin. All were negative in Bitter's arabinose and rhamnose and in Stern's glycerin-fuchsin bouillon. A heavy precipitate occurred in d-tartrate medium. Gelatin was not liquified. The Voges-Proskauer reaction was negative and litmus milk was rendered slightly alkaline. The strains varied in their behaviour towards lead acetate, the Yorkhill and the standard Kunzendorf culture both blackening this substance within twenty-four hours while the American strain completely failed to do so even after a week. Behaviour towards lead acetate is considered of importance in distinguishing between the American and Kunzendorf types, since the latter forms $\mathrm{H}_{2} \mathrm{~S}$ while the former does not. According to Bergey (1939) b. suipestifer typically ferments dulcite and dextrin with the production of acid and gas. The culture in the present case as well as the two Lister Institute strains failed to do so even after incubation for one week. It is well recognized, however, that in the Salmonella group there is a marked tendency to cultural variations, the serological characters being more stable (Kauffmann, 1934, 1935-36; Havens, 1935). Serological tests were then undertaken to complete the identification of the Yorkhill organism. That it possessed the $\mathrm{O}$ (somatic) antigens VI and VII of the paratyphosus C group was proved by the fact that an $\mathrm{O}$ antiserum prepared from a standard Lister Institute strain of b. paratyphosus $\mathrm{C}$ agglutinated it practically to titre. These $\mathrm{O}$ antigens are shared in common by b. paratyphosus $\mathrm{C}$ and by both the American and European strains of $b$. suipestifer. The $H$ (flagellar) antigenic formula of the last two differs in that the American strain is biphasic possessing the specific flagellar fraction $\mathrm{c}$ in addition to the non-specific fractions $1,3,4,5$, while the Kunzendorf variant is monophasic, having an identical non-specific antigenic formula, but lacking the specific $\mathrm{c}$ fraction. Antisera prepared in rabbits to the standard American and European suipestifer strains and to the unknown Yorkhill organism, agglutinated reciprocally all three cultures to approximately the same high titre, showing that all had common flagellar antigens. The next step in identification was the absorption of all the non-specific agglutinins from a suipestifer antiserum. The unknown Yorkhill organism, if monophasic, would then fail to agglutinate. Accordingly a heavy Kunzendorf growth was used to exhaust an antiserum to the American suipestifer strain, when the titre for the Yorkhill as well as for the stock Kunzendorf culture dropped from 
$1 / 25,000$ to less than $1 / 400$. On the other hand, the American strain was still agglutinated in high dilution by virtue of its unabsorbed specific flagellar fraction c. The Yorkhill organism was thus found to be monophasic, its serological as well as its main biological reactions corresponding with those of $b$. suipestifer var. Kunzendorf. The monophasic nature of the Yorkhill culture was confirmed by repeated testing, using on each occasion a number of isolated colonies from several plates. The toxicity of the strain was determined by rabbit inoculation. An animal weighing $2 \cdot 3 \mathrm{kgm}$. survived the intravenous inoculation of $1 / 100$ of a fresh agar culture suspended in saline, but $1 / 10$ of a culture killed a $3 \mathrm{kgm}$. animal within twenty-four hours. A heavy pure growth of the infecting organism was recovered from the heart blood, spleen and bile, and the intestinal flora at different levels consisted almost entirely of suipestifer colonies.

\section{Discussion}

Recognition of the pathogenicity for human beings of the suipestifer group of organisms dates largely from the last war when such infections were rife in eastern Europe, producing mainly paratyphoid-like disease. Originally $b$. suipestifer was considered pathogenic only for pigs in which it was thought to cause swine fever. It is now known, however, that here it plays the part merely of a secondary invader, the actual etiological agent being a filterable virus.

White (1926) classified suipestifer strains into four main types (a) American, (b) Eastern (Hirschfeld), (c) Western European, (d) Glässer-Voldagsen. Topley and Wilson (1936) regard the Glässer and Voldagsen strains as distinct although both are included in the 'Ferkeltyphus' bacilli of the German literature which produce typhoid-like disease in pigs, but so far as is known do not infect man. Within recent years human cases of suipestifer infection have been recorded in many parts of the world, occurring both in epidemic and sporadic form. Smith (1934) ranks the European type of b. suipestifer fifth in order of frequency among the Salmonella strains causing sporadic infections in this country. This is the most widespread suipestifer strain according to Boycott and McNee (1936), who state that only seven proved human infections with the American type are on record and that no certain infection with the Eastern (b. paratyphosus C) strain has occurred in Britain. In epidemics the type of disease produced is acute gastro-enteritis, diverse articles of food having been incriminated as the source of infection. Large outbreaks are reported by Schnitter (1927), Stewart and Litterer (1927); the occurrence of smaller epidemics is mentioned by Boller (1930) and Harvey (1937). In sporadic cases the organism may cause a gastro-intestinal type of disease simulating an enteric infection, or it may invade the blood-stream producing septicaemia with metastatic abscesses.

It appears from a survey of the literature that children are more susceptible than adults to suipestifer infection. White (1929), in reviewing the subject, states 'A disproportionately large number of the cases (of European suipestifer infection), especially those of abscess formation, occurred in infants and children; and most of those among adults are referable to the period of the War or the immediately succeeding years.' Nabarro et al. (1929) also regard the European strain as particularly apt to attack young children and to occur as frequently in localized disease as in general febrile infection and gastro-enteritis. Harvey (1937), reviewing a series of seventy-one suipestifer infections, mostly collected 
from the literature, found the majority of sporadic cases occurring in the first decade. Similarly of the twenty-eight cases reported by Ravitch and Washington (1937) almost all were in children. There is considerable diversity in the lesions produced by b. suipestifer. The typhoid-like clinical course is exemplified by the cases of Kuttner and Zepp (1932). Urinary excretion was observed by Rau (1932) in an infant with septicaemia. Gouley and Israel (1934) saw what appeared to be a true endocarditis arising during the course of a suipestifer septicaemia. B. suipestifer in addition to pneumococcus was cultured by Steuer (1937) from an empyema in a child aged three years. A pure growth of the former organism was recovered from an abscess which developed later in the thigh muscles. Clifton and Werner (1938) isolated a suipestifer strain from a sub-dural abscess. In the case of Ravitch and Washington this organism was grown from the cerebro-spinal fluid, but was thought by the authors to be derived actually from blood present in the specimen as the blood culture was positive. Whilst these examples serve to illustrate the protean nature of suipestifer infection, one particular lesion appears to predominate, especially in infants, i.e. septic arthritis. In Harvey's series skeletal disease was present in fifteen cases (approximately 20 per cent.) of which seven were infants in the first year of life. It is difficult to explain this localization, since trauma to the bones and joints would be less likely to occur in infancy than in later life. In fact, no history of injury is given in most of the recorded cases, the joint swelling having appeared insidiously. A number of cases which closely resemble that here recorded are reported in the literature. Thus Langwill (1921) records septicaemia in an infant with arthritis of the left shoulder joint, pus from which yielded an organism of the Salmonella group agglutinated by paratyphosus $\mathrm{C}$ antiserum.

Nabarro et al. saw infection of both shoulder joints in a female infant aged eight months, and isolated from the pus a typical American suipestifer strain. A joint lesion due to the European type in a child is also mentioned in their paper. The case reported by Bosch (1929) was an eleven-months-old infant with pus in the right knee-joint. Suipestifer infection in twins is described by Van Creveld and Ruys (1933). The first was admitted to hospital with a respiratory infection, and appeared at autopsy to have an ordinary bronchopneumonia with fibrinous pleurisy. Pneumonia was also diagnosed in the second twin who developed otitis media while in hospital. Later, in the course of the disease, the right knee suddenly swelled and a suipestifer strain was isolated from the blood-stained pus obtained by paracentesis, and also from the faeces. Tur and Gartoch (1934) observed a young infant whose mother died of a typhoid-like disease eighteen days post-partum. The child developed successively swelling of the right hand, right knee-joint, right shoulder and left ankle, in addition to a spreading broncho-pneumonia. A suipestifer strain was recovered from pus in the shoulder joint and from the faeces. Death occurred at the age of thirty-three days. A suppurative lesion affecting the right shoulder joint is recorded by Teveli (1935) in a child aged fifteen months who had pneumonia in addition. . The sputum, however, was negative for the Salmonella organism. The case of Gajzagó and Göttche (1935) bears a close similarity to the one here reported, since the lesion recorded by these authors, which also affected the shoulder in a young infant, was probably periarticular and not actually within the joint cavity. On x-ray examination the diaphysis of the humerus in their patient showed rarefaction 
thus suggesting the presence of an associated osteomyelitis. The rare condition of spinal osteomyelitis in the course of a suipestifer infection was seen in a child by Harvey (1937).

It is of interest that in the present case as well as in those of Teveli, Tur and Gartoch, Van Creveld and Ruys, pneumonia was associated with the articular lesion. It is not clear if the pulmonary lesion in the instances mentioned was actually of suipestifer origin, since no bacteriological data are given with regard to the lungs, except in Teveli's patient in whom the sputum was negative for $b$. suipestifer. In the present case cultures were not made from the lungs since the pneumonia appeared to be of ordinary suppurative type. The frequency of pulmonary complications in association with other lesions due to b. suipestifer is, however, emphasized by various writers.

Harvey found pulmonary or pleural involvement in one-third of the cases in his series. Cole and Nalls (1938) give a similar figure, and Haynes and Meiks (1933) consider the pulmonary type of suipestifer infection second only in frequency to the intestinal. A pure growth of b. suipestifer was actually isolated by Bullowa (1928) from an adult case which was clinically one of typical lobar pneumonia, and Harvey states that in six instances the diagnosis of a suipestifer lung infection was confirmed bacteriologically.

There appear to be grounds, therefore, for believing that pneumonia, when it occurs in the course of a suipestifer infection, is actually due to this organism.

Whilst it must be assumed that in suipestifer, as in other Salmonella infections, the portal of entry is the digestive tract, it is of interest that frequently no intestinal lesion is found at autopsy. This was true in the present case and in those of other investigators. Thus Cole and Nalls state that there is generally no morbid change in the gastro-intestinal tract and Boycott and McNee are of the opinion that intestinal ulcers are rare in suipestifer infections. Further, this organism is rarely isolated from the faeces. For example, in the series reported by Kuttner and Zepp it was recovered from the faeces of only one patient in seven, though repeated investigations were carried out in the majority. Other writers confirm the fact that faeces examination is generally negative, though blood cultures are frequently positive. As with other Salmonella strains the organism may enter by way of the pharynx, as suggested by Guerra et al. (1940). These authors instance the case of a child with a Salmonella septicaemia which began as a severe rhinopharyngitis followed by bilateral suppurative otitis media. The fact that the present patient also had a bilateral otitis and a history of a 'cold' before the onset of the joint swelling, lends support to the hypothesis of a rhinopharyngeal portal of entry.

The source of suipestifer, like other sporadic Salmonella infections, is generally quite obscure as in the present instance. There is seldom a history of association with another patient, and only occasionally in the literature is there any history of contact with diseased pigs. Proof that these animals are not necessarily concerned in the spread of such infections is afforded by the experience of Neukirch (1918) who observed cases due to b. paratyphosus $C$ in Turkey where the rearing of pigs is prohibited by the Mohammedan creed. It must be assumed that the suipestifer bacillus is ingested in contaminated food. 
The high morbidity rate in children may depend on the fact that as compared with adults they have less resistance to infection and hence may be susceptible to a relatively small inoculum.

\section{Summary}

A case is described of suipestifer infection in an infant with the characteristic localization in relation to a joint. The literature on suipestifer infection in children is shortly reviewed.

Thanks are due to Mr. Matthew White, F.R.C.S., for permission to make use of the case record.

\section{REFERENCES}

Bergey, D. H., et al. (1939). Manual of determinative bacteriology, fifth edition, London, 441. Boller, E. (1930). Schweiz. med. Wschr., 60, 1035.

Bosch, W. G. (1929). Geneesk. Tijdschr. Ned.-Ind., 69, 42.

Boycott, J., and McNee, J. W. (1936). Lancet, 2, 741.

Bullowa, J. G. M. (1928). Med. Clin. N. Amer., 12, 691.

Clifton, W. M., and Werner, M. (1938). Amer. J. Dis. Child., 55, 553.

Cole, D. B., and Nalls, W. L. (1938). J. Lab. clin. Med., 23, 1223.

Gajzágó, D., and Göttche, O. (1935). Amer. J. Dis. Child., 49, 1270.

Gouley, B. A., and Israel, S. L. (1934). Arch. intern. Med., 53, 699.

Guerra, A. R., Peluffo, E., and Aleppo, P. L. (1940), Bonaba and others. Estudios sobre la Etiología Infecciosa de las Diarreas Infantiles, Montevideo, 91.

Guthrie, K. J., and Montgomery, G. L. (1939). J. Path. Bact., 49, 393.

Harvey, A. M. (1937). Arch. intern. Med., 59, 118.

Havens, L. C. (1935). The bacteriology of typhoid, salmonella and dysentery infections and carrier states, New York, Commonwealth Fund.

Haynes, E., and Meiks, L. T. (1933). Amer. J. Dis. Child., 46, 1054.

Kauffmann, F. (1934). Ergebn. Hyg. Bakt., 15, 219. (1935-36). Z. Hyg. Infektkr., 117, 431.

Kuttner, A. G., and Zepp, H. D. (1932). Johns Hopk. Hosp. Bull., 51, 373.

Langwill, A. (1921). Lancet, 2, 1158.

Nabarro, D., White, P. B., Dyke, S. C., and Scott, W. M. (1929). Ibid., 2, 868.

Neukirch, P. (1918). Z. Hyg. Infektkr., 85, 103.

Rau, H. (1932). Z. Kinderheilk., 52, 510.

Ravitch, M. M., and Washington, J. A. (1937). J. Amer. med. Ass., 109, 1122.

Schnitter (1927). Münch. med. Wschr., 74, 1011.

Smith, J. (1934). J. Hyg., Camb., 34, 351.

Steuer, W. (1937). Zbl. Bakt., Abt. 1, Orig., 140, Beiheft, 67.

Stewart, H. C., and Litterer, W. (1927). J. Amer. med. Ass., 89, 1584.

Teveli, Z. (1935). Arch. Kinderheilk., 104, 41.

Topley, W. W. C., and Wilson, G. S. (1936). The principles of bacteriology and immunity, London, 557.

Tur, A. F., and Gartoch, O. O. (1934). Z. Kinderheilk., 56, 696.

Van Creveld, S., and Ruys, A. C. (1933). Ibid., 54, 725.

White, P. B. (1926). Medical Research Council special reports No. 103, London.

- (1929). Medical Research Council. A system of bacteriology, London, 4, 129. 